NASA-TM-112133

\section{Microwave Backscatter and Attenuation Dependence on Leaf Area Index for Flooded Rice Fields}

Stephen L. Durden, Leslie A. Morrissey, and Gerald P. Livingston

\begin{abstract}
Wetlands are important for their role in global climate as a source of methane and other reduced trace gases. As part of an effort to determine whether radar is suitable for wetland vegetation monitoring, we have studied the dependence of microwave backscatter and attenuation on leaf area index (LAI) for flooded rice fields. We find that the radar return from a flooded rice field does show dependence on LAI. In particular, the $C$-band VV cross section per unit area decreases with increasing LAI. A simple model for scattering from rice fields is derived and fit to the observed $\mathrm{HH}$ and $\mathrm{VV}$ data. The model fit provides insight into the relation of backscatter to LAI and is also used to calculate the canopy path attenuation as a function of LAI.
\end{abstract}

\section{INTRODUCTION}

Wetlands are important for their role in global climate as a source of methane and other reduced trace gases. Primary controlling factors

Manuscript received May 3, 1994.; revised December 20, 1994. This work was performed by the Jet Propulsion Laboratory, California Institute of Technology, under contract with the National Aeronautics and Space Administration (NASA), and by NASA Ames Research Center. Funding was provided by the NASA Polar and Ecological Processes and Modeling Programs.

S. L. Durden is with the Jet Propulsion Laboratory, California Institute of Technology, Pasadena, CA 91109 USA.

L. A. Morrissey and G. P. Livingston are with the Johnson Controls World Services, NASA Ames Research Center, Moffett Field, CA 94035 USA.

IEEE Log Number 9409909. for methane emission include vegetation characteristics and presence of surface water [1]. With the availability of ERS-1 and JERS-1 and the planned flight of RADARSAT, imaging radar is a candidate for remote sensing of these controlling factors. It has been shown that radar is sensitive to the biomass of woody vegetation (e.g., trees) for low to moderate biomass [2]. It has also been shown that radar is sensitive to surface water under trees [3], at least at longer wavelengths (e.g., $L$-band). Most measurements of herbaceous vegetation (e.g., grasses) by radar have focused on agricultural crops without surface water present. These studies have demonstrated sensitivity to leaf area index (LAI) [4], biomass, and canopy height [5]. Radar's response to herbaceous vegetation in wetlands is less well documented. Ott et al. [6] presented evidence that multipolarization SAR can distinguish wetlands vegetation in a mid-latitude setting, while [7] found similar results for a tropical setting. Reference [8] demonstrated that ERS-1 SAR backscatter is sensitive to water table position and LAI for herbaceous Arctic tundra. Here, we examine the radar response to LAI in flooded rice fields as a step toward understanding this response in natural herbaceous wetlands.

\section{MEASUREMENTS}

The data examined here were acquired in 19 flooded rice fields at mid growing season in central California near the town of Colusa in July 1991. At each field in situ measurements were made at one or two sample plots located approximately $10 \mathrm{~m}$ from the edge of the field. Each sample plot had an area of roughly $500 \mathrm{~cm}^{2}$. LAI was measured by harvesting the vegetation, measuring the total green leaf area, and normalizing by the area of the sample plot. Stem number density was found by counting the total number of stems in the plot and normalizing by the plot area. Mean plant height was also computed. 
TABLE I

Rice Field Vegetation Parameters and Radar Cross Sections per Untt ARea

\begin{tabular}{|c|c|c|c|c|c|c|}
\hline LAI & PTant Ht. $(\mathrm{cm})$ & Stem $/ \mathrm{m}^{2}$ & LVV (dB) & LHH (dB) & $\mathrm{CW}(\mathrm{dB})$ & CHH (dB) \\
\hline $1 . \overline{8}$ & 70 & 551 & -15.4 & -10.8 & .8 .2 & -3.1 \\
\hline 1.8 & 56 & 561 & -16.6 & .17 .1 &.$\$ .0$ & -3.4 \\
\hline 1.9 & 66 & 502 & -14.2 & -14.8 & -5.0 & -3.4 \\
\hline 2.1 & 65 & 650 & -11.7 & -13.7 & -8.0 & -3.4 \\
\hline 2.3 & 75 & 571 & -10.2 & -12.1 & -6.0 & -3.0 \\
\hline 2.6 & 84 & 571 & -7.0 & -9.7 & -5.6 & -2.6 \\
\hline 3.0 & 75 & 984 & .12 .8 & .14 .8 & -8.1 & -1.9 \\
\hline 3.0 & 72 & 719 & .8 .3 & -10.2 & -5.1 & -2.8 \\
\hline 3.1 & 74 & 925 & -11.7 & .11 .6 & -12.5 & -3.2 \\
\hline 3.1 & 76 & 620 & -3.8 & -4.7 & -7.9 & -2.7 \\
\hline 3.2 & 62 & 778 & -8.7 & -12.2 & -4.8 & -2.5 \\
\hline 3.4 & 66 & 955 & .12 .7 & -14.7 & -8.6 & -2.8 \\
\hline 3.4 & 69 & 778 & -14.7 & -16.6 & -6.8 & -3.5 \\
\hline 3.7 & 67 & 738 & -13.8 & -15.6 & -7.4 & -3.6 \\
\hline 3.8 & 64 & 847 & 0.2 & 0.2 & -10.9 & -3.3 \\
\hline 3.8 & 81 & 1103 & -10.2 & -8.9 & -11.9 & -3.6 \\
\hline 4.0 & 76 & 787 & -12.4 & -13.8 & -10.9 & -3.4 \\
\hline 4.5 & 76 & 768 & -12.1 & -11.3 & -11.6 & -4.5 \\
\hline 3.1 & 66 & 1378 & 0.6 & 2.6 & -9.3 & -3.5 \\
\hline
\end{tabular}

Table I shows LAI, plant height, and number density for all 19 fields. The LAI ranges between 1.6 and 5.1. All fields had been planted at essentially the same time and were therefore at roughly the same phenological stage. The variability in LAI between fields is primarily caused by variability in number density. LAI and plant height are not correlated at the $1 \%$ level, while LAI and number density are correlated $(r=0.77)$. All plants were quite green, indicating a high water content.

Radar measurements of the rice fields were acquired with the NASA/JPL DC-8 AIRSAR [9]. This is an airborne synthetic aperture radar operating at UHF $(68 \mathrm{~cm}), L$-band $(24 \mathrm{~cm})$, and $C$-band $(5.6$ $\mathrm{cm})$. Because of severe interference at UHF, only $L$-band and $C$-band data are used in this study. Several comer reflectors were deployed in the area, and these were used in verifying the absolute calibration. The incidence angle for all 19 rice fields was approximately $50^{\circ}$. Radar parameters for each field were found by averaging the fourlook radar data from several hundred pixels within the field. The like-polarized cross sections at $L$ - and $C$-bands are shown in Table I. The only cross section that is correlated with LAI at the $1 \%$ significance level is the $C$-band VV cross section $(r=-0.69)$. The reason for the negative correlation at $C$-band will be discussed in Section III. We also examined the HV cross section, the HH-VV correlation coefficient, and the HH-VV phase difference at both $L$ and $C$-bands. Of these six parameters, only the $C$-band correlation coefficient shows dependence on LAI, decreasing from around 0.3 at low LAI to 0.1 at high LAI $(r=-0.83)$. The $\mathrm{HH}-\mathrm{VV}$ phase difference is not correlated with LAI and varies between $60^{\circ}$ and $150^{\circ}$ at $L$-band and between $0^{\circ}$ and $80^{\circ}$ at $C$-band.

\section{MODELING}

We model a flooded rice field as a single layer of discrete scatterers over a reflecting surface, using the Distorted Born Approximation [10]-[13]. The water surface is assumed flat so that there is no direct backscattering from it; it acts only as a specular reflector. In other situations, it is necessary that the surface backscatter be included, as done by [5] for $X$-band scattering from wheat in rough soil. For rice, the scattered field is the sum of direct, direct-reflected, and reflected components. These components are illustrated in Fig. 1 and have been discussed widely in the literature. The direct component is often referred to as volume scattering, while the direct-reflected term is often referred to as double-bounce scattering. The reflected component is assumed to be small relative to the direct term because of attenuation due to an extra round trip through the canopy and due to two reflections from the water surface. From this point on we

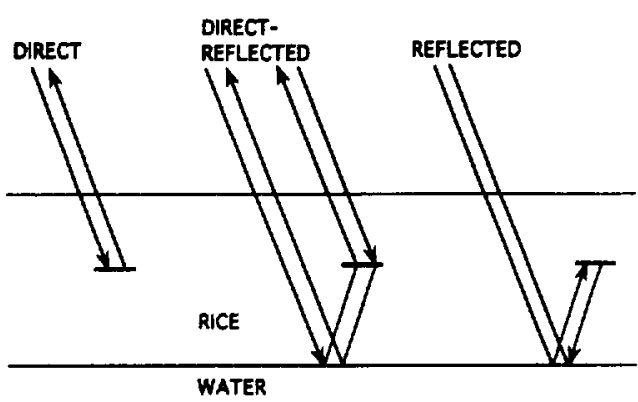

Fig. 1. Illustration of the direct, direct-reflected, and reflected components in scattering from a layer of rice over a water surface.

neglect it and consider the scattered field to be a sum of the direct $(d)$ and direct-reflected $(d r)$ terms.

Assuming a single particle within the medium, having a scattering matrix $S$, the direct and direct-reflected scattered fields are

$$
\begin{aligned}
& E_{d}^{A B}=S_{d}^{A B} \exp \left(i\left(K_{A}+K_{B}\right) t\right) \\
& E_{d r}^{A B}=\left(S_{d r}^{A B} R_{A}+S_{d r}^{B A} R_{B}\right) \exp \left(i\left(K_{A}+K_{B}\right) T\right)
\end{aligned}
$$

where $S$ is the scattering matrix, $R$ is the Fresnel reflection coefficient, $T$ is the total distance the wave travels from the top of the medium to the surface, $t$ is the distance traveled by the wave from the top of the medium to the particle, and $K^{-}$is the propagation constant for the medium, which can be found from the optical theorem [10]. $A$ and $B$ denote the polarizations of the scattered and incident waves, respectively, and may be $H$ or $V$. Note that the scattered fields are spherical waves. Also note that depolarization due to propagation through the medium has been neglected. Second-order statistics of the scattered field are found by averaging over the depth of the medium $h$ and over the particle size and orientation distributions. For the like polarization case the cross section per unit area can be written as

$$
\begin{aligned}
\sigma^{\circ}= & \eta_{d} \cos \theta(1-\exp (-2 h / \cos \theta)) / 2 \alpha \\
& +\eta_{d r} h|R|^{2} \exp (-2 \alpha h / \cos \theta)
\end{aligned}
$$

where $\eta$ is the cross section per unit volume, $\alpha$ is the attenuation coefficient, $\theta$ is the incidence angle measured from nadir. In (3) the first term is the direct component, while the second term is the direct-reflected component. As was pointed out in Section II, the primary reason for the observed variability in LAI is variability in the number of rice plant stems per unit area. Since LAI, $\eta$, and $\alpha$ are all proportional to the number of scatterers per unit volume $n_{o}$, we assume that both $\eta$ and $\alpha$ are proportional to LAI. Using these assumed dependences

$$
\begin{aligned}
\sigma^{\circ}= & \cos \theta\left(1-\exp \left(-2 c_{2} \mathrm{LAI} / \cos \theta\right)\right) c_{1} / 2 c_{2} \\
& +c_{3}|R|^{2} \mathrm{LAI} \exp \left(-2 c_{2} L A I / \cos \theta\right)
\end{aligned}
$$

and $\eta_{d}=c_{1} \mathrm{LAI} / h, \eta_{d r}=c_{3} \mathrm{LAI} / h$, and $\alpha=c_{2} \mathrm{LAI} / h$. Note that if we were trying to model the time dependence of radar return from a single rice plot, this model would not be appropriate. The LAI variation would be related to changes in leaf size rather than number density. Neither $\eta$ nor $\alpha$ are linearly related to leaf size, so a different model would be needed.

The model described by (4) was fit to the $\mathrm{HH}$ and $\mathrm{VV} \sigma^{\circ}$ measurements using nonlinear least squares. The fit was performed using the cross section expressed in decibels so that large and small cross sections were weighted equally. The value of $|R|^{2}$ in (4) was calculated at each polarization using the dielectric constant for pure water [14]. The incidence angle was $50^{\circ}$. Table II shows the resulting model coefficients and associated rms error. We have used these coefficients to calculate the direct and direct-reflected components 


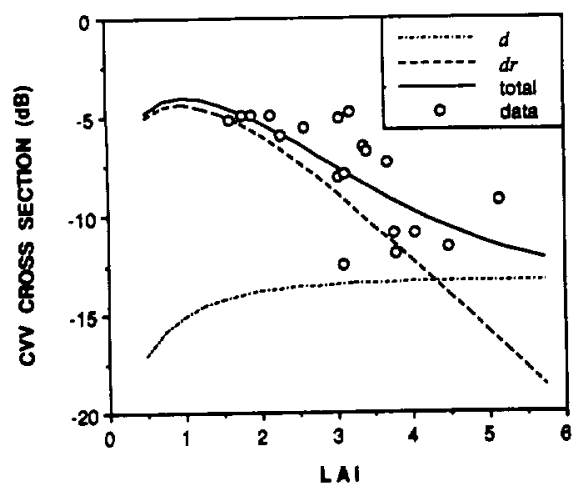

Fig. 2. Calculated dependence of $\mathrm{CVV} \sigma^{\circ}$ on LAI for $50^{\circ}$ incidence. Shown are the total cross section and the direct $(d)$ and direct-reflected $(d r)$ components. Also shown are the observed CVV data.

TABLE II

COEFFICIENTS Resulting FROM Model FIT

\begin{tabular}{ccccc} 
& $c_{1}$ & $c_{2}$ & $c_{3}$ & RMS ERROR (dB) \\
\hline IVV & 0.00 & 0.00 & 0.06 & $\mathbf{4 . 2 2}$ \\
LHH & 0.00 & 0.00 & 0.03 & 4.76 \\
CVV & 0.05 & 0.34 & 2.15 & 1.87 \\
CHH & $\mathbf{0 . 0 0}$ & 0.12 & 0.70 & 0.44 \\
\hline
\end{tabular}

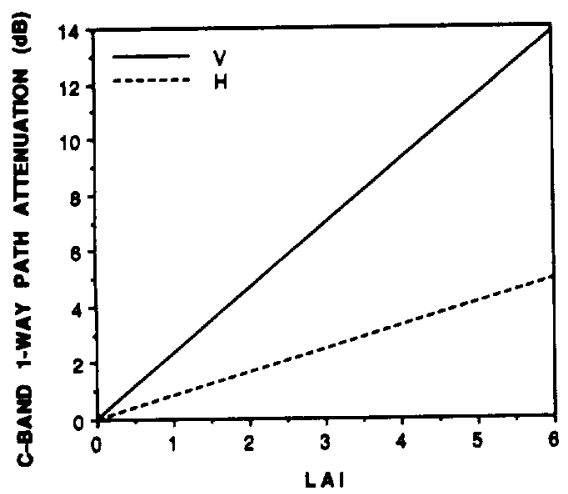

Fig. 3. $C$-band one-way canopy path attenuation at $50^{\circ}$ incidence angle for $\mathrm{H}$ and $\mathrm{V}$ polarizations, as found from nonlinear least squares fit of model to data.

and the total cross section as a function of LAI for LVV, LHH, $\mathrm{CVV}$, and $\mathrm{CHH}$. The direct-reflected term is greater than the direct term in all cases except for CVV with LAI greater than 4.3. Results for the CVV case are shown in Fig. 2, along with the observed CVV data. The increasing importance of the direct term is the likely explanation for the decrease in the $\mathrm{HH}-\mathrm{VV}$ correlation coefficient with LAI, noted in Section II. In Table II, the $c_{2}$ coefficient is very close to zero at $L$-band, implying that the $L$-band attenuation is small. In contrast, the $c_{2}$ coefficients for $C$-band are nonzero, implying a higher attenuation at $C$-band than at $L$-band, as would be expected. The one-way path attenuation in decibels at the $50^{\circ}$ incidence angle is $4.343 c_{2}$ LAI $50^{\circ}$. Fig. 3 shows the calculated path attenuation versus LAI for $C$-band. The $\mathrm{V}$ polarization attenuation is larger than that for $H$ polarization because of the vertical orientation of the rice blades. While no independent rice canopy attenuation measurements are known to the authors, the attenuations derived here are similar to attenuation measurements in other agricultural crops with comparable leaf area index [15].

These modeling results provide understanding of why the CVV backscatter decreases with increasing LAI. The model fit indicates that CVV backscatter from flooded rice fields is dominated by the direct-reflected term for LAI up to 4.3. As can be seen in (3), this term increases linearly with the cross section per unit volume $\eta_{d r}$ but decreases exponentially with the attenuation coefficient $\alpha$. Based on the assumed dependences of these parameters on LAI in (4), $\sigma^{\circ}$ will increase with LAI for small path attenuations and decrease with LAI for large path attenuations. This effect can be seen in the model calculation in Fig. 2. For LAI less than one, $\sigma^{\circ}$ increases with LAI. For LAI greater than one, $\sigma^{\circ}$ decreases with LAI. Note that these results are different from the case of scattering from forests where it has often been noted that radar backscatter increases with increasing vegetation, eventually saturating (e.g., [2]). In that case, the forest floor is typically a rough soil surface, so the direct rather than directreflected term should dominate. As can be seen in the direct term in (3), for large attenuations the exponential becomes small, making $\sigma^{\circ}$ proportional to the ratio of the $\eta_{d}$ to $\alpha$. In this case, an increase in vegetation increases both $\eta_{d}$ and $\alpha$, causing their ratio to remain nearly constant. This behavior can be seen in the direct term in Fig. 2 .

\section{CONCLUSIONS}

To address the problem of relating radar backscatter to LAI in herbaceous wetlands, this study took advantage of an agricultural setting in which LAI variability was caused by variability in the stem number density. We found that $C$-band, VV polarization radar backscatter decreased with increasing LAI over the observed range of LAI. A simple model was fit to the observed data, and the resulting fit showed that the decrease in CVV backscatter with LAI is related to the domination of direct-reflected backscatter over the direct component. Model calculations suggested that at very low LAI the radar backscatter should increase with LAI. This being the case, it is possible that very low and very high LAI sites could have the same radar backscatter cross section, making differentiation of a wide range of LAI on a single date using CVV radar data alone impossible. If this is the case, it may be possible to resolve the ambiguity by using a CVV time series to monitor LAI, for example, over a growing season. Also, use of additional data, such as radar data at a different frequency or incidence angle, might resolve the ambiguity.

\section{REFERENCES}

[1] L. A. Morrissey and G. P. Livingston, "Methane flux from tundra ecosystems in arctic Alaska," J. Geophys. Res., vol. 97, pp. 16661-16670, 1992.

[2] M. C. Dobson, F. T. Ulaby, T. LeToan, A. Beaudoin, and E. S. Kasischke, "Dependence of radar backscatter on coniferous forest biomass," IEEE Trans. Geosci. Remote Sensing, vol. 30., pp. 412-415, 1992.

[3] L. L. Hess, J. M. Melack, and D. S. Simonett, "Radar detection of flooding beneath the forest canopy: A review," Int. J. Remote Sensing, vol. 11, pp. 1313-1325, 1990.

[4] F. T. Ulaby, C. T. Allen, G. Eger, and E. Kanemasu, "Relating the microwave backscatter coefficient to leaf area index," Remote Sensing Environ., vol. 14, pp. 113-133, 1984.

[5] B. A. M. Bouman, "Crop parameter estimation from ground-based Xband ( $3 \mathrm{~cm}$ wave) radar backscattering data," Remote Sensing Environ., vol. 37, pp. 193-206, 1991.

[6] J. S. Ott, E. S. Kasischke, N. H. French, M. F. Gross, and V. Klemis, "Evaluation of multichannel SAR data for analysis of a mid-Atlantic coastal wetland ecosystem," in Proc. IGARSS'90, College Park, MD, 1990, pp. 453-456.

[7] K. O. Pope, J. M. Reybenayas, and J. F. Paris, "Radar remote sensing of forest and wetland ecosystems in the Central American tropics," Remote Sensing Environ., vol. 48, pp. 205-219, 1994.

[8] L. A. Morrissey, G. P. Livingston, and S. L. Durden, "Use of SAR in regional methane exchange studies," Int. J. Remote Sensing, vol. 15, no. 6. pp. $1337-1342,1994$.

[9] A. Freeman, Y. Shen, and C. L. Werner, "Polarimetric SAR calibration experiment using active radar calibrators," IEEE Trans. Geosci. Remote Sensing, vol. 28, pp. 224-240, 1990. 
[10] L. Tsang, J. A. Kong, and R. T. Shin, Theory of Microwave Remote Sensing. New York: Wiley, 1985.

[11] R. H. Lang and J. S. Sidhu, "Electromagnetic backscattering from a layer of vegetation: A discrete approach," IEEE Trans. Geosci. Remote Sensing, vol. GRS-21, pp. 62-71, 1983.

[12] R. H. Lang and H. A. Saleh, "Microwave inversion of leaf area and inclination angle distributions from backscattered data," IEEE Trans. Geosci. Remote Sensing, vol. GRS-23, pp. 685-694, 1985.

[13] N. S. Chauhan, R. H. Lang, and J. K. Ranson, "Radar modeling of a boreal forest," IEEE Trans. Geosci. Remote Sensing, vol. 29, pp. 627-638. 1991

[14] P. S. Ray, "Broadband complex refractive indices of ice and water," Appl. Opt., vol. 11, no. 8, pp. 1836-1844, 1972.

[15] F. T. Ulaby and E. A. Wilson, "Microwave attenuation properties of vegetation canopies," IEEE Trans. Geosci. Remote Sensing, vol. GRS23. pp. 746-753, 1985. 